\title{
Comparison of Two Methods for Screening TCR VB Biases of T1DM Patients: Silver Staining for Polyacrylamide and Ethidium Bromide Staining for Agarose Gel Electrophoresis
}

\author{
Jianwei Zhou ${ }^{1, *}$, Cui Kong ${ }^{2}$, Bo Ban ${ }^{3}$, Lin Sun ${ }^{3}$, Cuiyun Dou ${ }^{1}$, Zhaocai Zhang ${ }^{1}$ \\ ${ }^{1}$ Clinic Laboratory, the Affiliated Hospital of Jining Medical College, Jining, Shandong Province, China \\ ${ }^{2}$ Department of Cardiovascular Disease, the Affiliated Hospital of Jining Medical College, Jining, Shandong Province, China \\ ${ }^{3}$ Department of Endocrinology, the Affiliated Hospital of Jining Medical College, Jining, Shandong Province, China \\ *Corresponding author: immunolife@126.com
}

Received September 30, 2013; Revised October 25, 2013; Accepted November 19, 2013

\begin{abstract}
Objective: To explore a fast and sensitive method for screening T cell receptor (TCR) biases through comparison of silver staining for polyacrylamide gel electrophoresis and ethidium bromide staining for agarose gel electrophoresis. Methods: The lymphocytes were isolated from peripheral blood which collected from ten patients with T1DM; the total RNA was extracted followed by transformed to cDNA, and then electrophoresis was carried out on polyacrylamide and agarose gel simultaneously, finally stained with silver and ethidium bromide dying respectively. Results: The pictures of TCR V $\beta$ biases detected with silver staining for polyacrylamide gel electrophoresis were very similar to that with ethidium brominde staining for agarose gel electrophoresis, V $\beta 7$, V $\beta 11$ and V $\beta 17$ were the mainly biased genes with higher frequencies, 10 and V $\beta 13.2$ were the common genes which did not biased in all the ten patients. In some $\mathrm{V} \beta$ genes, the number of the bands detected with the former technique was more than that with the latter methods. So did the clarity of some bands. Conclusions: Silver staining for polyacrylamide gel electrophoresis is a fast, sensitive and safe method for screening the biases of TCR V $\beta$ genes.
\end{abstract}

Keywords: silver staining, polyacrylamide gel electrophoresis, ethidium bromide staining, agarose gel electrophoresis, $T$ cell receptor, typ1 a diabetes mellitus

Cite This Article: Jianwei Zhou, Cui Kong, Bo Ban, Lin Sun, Cuiyun Dou, and Zhaocai Zhang, "Comparison of two Methods for Screening TCR V $\beta$ Biases of T1DM Patients: Silver Staining for Polyacrylamide and Ethidium Bromide Staining for Agarose Gel Electrophoresis.” American Journal of Medical Sciences and Medicine 1, no. 6 (2013): 101-104. doi: 10.12691/ajmsm-1-6-1.

\section{Introduction}

Mature T cells express one of two types of TCR: a heterodimer of $\alpha$ and $\beta$ chains or $\gamma$ and $\delta$ chains. High expansions of $\alpha \beta$ T cells within the TCR repertoire have been shown to occur in various malignancies and immunological disorders, in addition to inflammatory and infectious diseases [1]. If biased TCR variable region (V) was associated with the pathogenic T-cell populations, the corresponding TCR elements could be targeted by TCRspecific immunotherapies, such as TCR peptide or T-cell vaccination [2,3]. For this reason, the studies of TCR V gene biases are attached much weight by researchers.

To date, the techniques, such as Southern blot analysis [4], staining with TCR V-gene-specific antibodies [5], PCR-ELISA [6], immuoscope spectratyping technique [7] and so on, were applied to detecting expansion of TCR V gene. More presently, the real-time florescence quantitative polymerase chain reaction (FQ-PCR) and DNA melting curve analysis technique was successfully modified and used to detecting skewness of TCR [8,9]. However, the above methods either need special instruments, or expensive reagents, or the procedure was too complicated to operate. Although there had been a method, ethidium brominde staining for agarose gel electrophoresis (which is called ethidium brominde staining technique in the following text), to screen the skewness of TCR, but its poisonousness of the dying limits its application. In this study, we used silver staining for polyacrylamide gel electrophoresis (which is called silver staining technique in the following text) and ethidium brominde technique to detect biases of TCR V $\beta$ of patients with type 1 diabetes mellitus (T1DM), and hope to ensure if the former method could replace the later one.

\section{Methodology}

\subsection{Subjects}

All the patients with T1DM were diagnosed according to the standard of WHO [10]. Peripheral blood 
lymphocytes (PBL) were collected from ten patients with T1DM and healthy volunteers, who were not treated with immunomodulating drugs in the previous six months prior to the study and were seronegative for markers of hepatitis viruses, HIV and other pathogenic infections. Excluded from the study were patients with tumors and immunological disorders. This study protocol was approved by the hospital Ethics Committee.

\subsection{Extraction of RNAs and Synthesis of the First cDNAs}

The sense primer, anti-sense primer and specific primers for 24 TCR V $\beta$ genes families (both of V $\beta 5$ and V $\beta 13$ include two subfamilies: V $\beta 5.1$ and V $\beta$ 5.2, V $\beta 13.1$ and V $\beta 13.2$ ) were previously described [1] and synthesized by the Guangzhou Daangene Corporation of China. A total of $5 \mathrm{ml}$ of blood were taken from each of T1DM patients and healthy volunteers respectively. PBLs were isolated by Fcoll-Hypaque density centrifugation. Using an Omega RNA extraction kit according to the manufacturer's instructions, total RNA was extracted, and $1 \mu \mathrm{g}$ total RNA was reverse transcribed with $250 \mathrm{pm}$ olig (dT), 200 U Moloney murine leukemia virus (M-MuLV) reverse transcriptase, and $2 \mu$ of $10 \mathrm{mM} \mathrm{dNTP} \operatorname{mix}$ (cDNA Synthesis Kit; MBI-Fermentas), in a total volume of $20 \mu \mathrm{l}$ (six reactions for every sample). The cDNA was stored at $-80^{\circ} \mathrm{C}$.

\subsection{Analysis of TCR VB Clonality with Two Methods}

Ethidium bromide staining for agarose gel: according to the previous study $[1,8,9], 8 \mu \mathrm{l}$ PCR products were added in $1.5 \%$ agarose gel followed by staining with ethidium bromide.

Silver staining for polyacrylamide gel electrophoresis: $2 \mu \mathrm{l}$ PCR products was spotted in $12 \%$ polyacrylamide gel with the thickness of 1 millimeter, and performed electrophoresis for $60 \mathrm{~min}$, and then stained with $0.2 \%$ silver nitrate for $30 \mathrm{~min}$, developed by $3 \%$ sodium hydroxide and $0.1 \%$ formaldehyde.

\section{Results}

On the $1.5 \%$ agarose gel stained with ethidium brominde, each family of TCR V $\beta$ genes of healthy volunteers only showed a comparatively clear band at the position of about $250 \mathrm{bp}$, and the picture of every $\mathrm{V} \beta$ gene was similar to that of V $\beta 1$ of patient- 1 (as shown as Figure 1 ), so the data was not shown. While for the patients with T1DM, except for most V $\beta$ genes exhibited single clear band at the predicted position, several showed two or more than two bands, or there were a few genes which exhibited more blurry bands, even looked disappear (Figure 1, only the picture of patient-1 was shown). Compared to healthy volunteers, except for V $\beta 8$, V $\beta 10$, $\mathrm{V} \beta 12, \mathrm{~V} \beta 13.2$ and $\mathrm{V} \beta 15$, the other $\mathrm{V} \beta$ genes showed more or less biased, and the mainly biased genes were $\mathrm{V} \beta 7$, $\mathrm{V} \beta 11$ and V $\beta 17$. Besides, the bands of V $\beta 18$ of patient- 1 , V $\beta 20$ of patient- 6 and -8 were found deficient or deleted (Table 1).

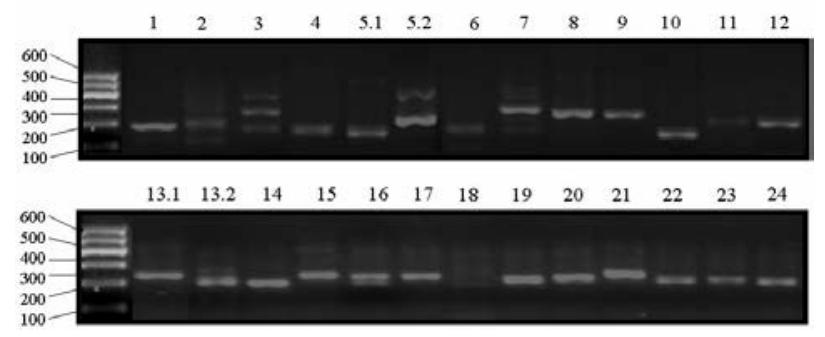

Figure 1. The pictures for 24 TCR V $\beta$ genes of TIDM patients- 1 on the $1.5 \%$ agarose gel by ethidium brominde staining

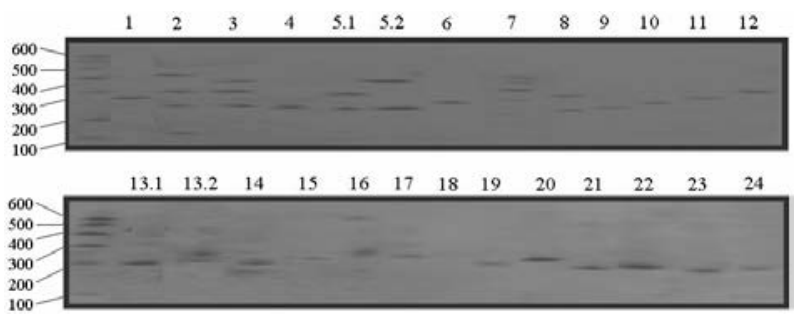

Figure 2. The pictures for 24 TCR V $\beta$ genes of TIDM patients- 1 on the $12 \%$ polyacrylamide gel by silver staining

\begin{tabular}{|c|c|c|c|c|c|c|c|c|c|c|}
\hline $\mathrm{V} \beta$ & P1 & P2 & P3 & $\mathrm{P} 4$ & P5 & P6 & P7 & P8 & P9 & P10 \\
\hline 1 & $\mathrm{~A}$ & $\mathrm{~A}$ & A & A & B & A & $\mathrm{A}$ & B & B & $\mathrm{A}$ \\
\hline 2 & B & A & A & B & A & A & A & A & A & B \\
\hline 3 & $\mathrm{C}$ & A & A & A & A & A & A & A & A & A \\
\hline 4 & A & A & A & A & A & A & A & A & A & A \\
\hline 5.1 & A & A & A & A & A & A & A & $\mathrm{C}$ & A & A \\
\hline 5.2 & B & A & A & A & A & A & A & A & A & A \\
\hline 6 & $\mathrm{~A}$ & A & A & A & A & A & C & A & A & A \\
\hline 8 & A & A & A & A & A & A & A & A & A & A \\
\hline 9 & A & A & B & A & A & A & A & A & A & A \\
\hline 10 & $\mathrm{~A}$ & A & A & A & A & A & A & A & A & A \\
\hline 11 & A & B & $\mathrm{C}$ & A & C & B & A & A & A & A \\
\hline 12 & A & A & A & A & A & A & A & A & A & A \\
\hline 13.1 & A & A & A & A & A & A & A & B & A & A \\
\hline 13.2 & A & A & A & A & A & A & A & A & A & A \\
\hline 14 & A & A & A & A & B & A & A & A & A & A \\
\hline 16 & A & A & A & A & C & A & A & A & A & A \\
\hline 17 & A & B & B & B & A & A & A & A & A & B \\
\hline 18 & $\Delta$ & A & A & A & A & A & B & A & A & A \\
\hline 19 & A & A & B & A & A & A & A & A & A & A \\
\hline 20 & A & A & A & A & A & $\boldsymbol{\Delta}$ & A & $\boldsymbol{\Delta}$ & A & A \\
\hline 21 & A & B & A & A & A & A & A & A & A & A \\
\hline 22 & A & A & A & A & A & A & A & A & B & A \\
\hline 23 & A & A & A & A & B & A & A & A & A & A \\
\hline 24 & A & A & A & A & $\mathrm{A}$ & A & A & B & A & A \\
\hline
\end{tabular}

A, B, C, D and E represented one, two, three, four and five band(s) respectively; $\boldsymbol{\Delta}$ represented the TCR V $\beta$ gene families which exhibited no band. 
Table 2. The biases of 24 TCR V $\beta$ genes in T1DM patients with polyacrylamide gel electrophoresis by silver staining

\begin{tabular}{|c|c|c|c|c|c|c|c|c|c|c|}
\hline $\mathrm{V} \beta$ & P1 & P2 & P3 & P4 & P5 & P6 & P7 & P8 & P9 & P10 \\
\hline 1 & $\mathrm{~A}$ & $\mathrm{~A}$ & A & A & B & $\mathrm{A}$ & A & B & $\mathrm{A}$ & $\mathrm{A}$ \\
\hline 2 & D & A & A & A & A & A & A & A & A & B \\
\hline 3 & C & A & A & A & A & A & A & A & A & A \\
\hline 4 & A & A & A & A & A & B & A & A & A & A \\
\hline 5.1 & B & A & A & A & A & A & A & $\mathrm{C}$ & A & A \\
\hline 5.2 & B & A & A & A & A & A & A & A & A & A \\
\hline 6 & A & A & A & A & A & A & D & A & A & A \\
\hline 7 & $\mathrm{E}$ & C & A & D & B & B & A & C & B & C \\
\hline 8 & B & A & A & A & A & A & A & A & A & A \\
\hline 9 & A & A & B & A & A & A & A & A & A & A \\
\hline 10 & A & A & A & A & A & A & A & A & A & A \\
\hline 11 & A & $\mathrm{C}$ & D & A & C & B & A & A & A & C \\
\hline 12 & A & A & $\mathrm{C}$ & A & A & A & A & A & A & A \\
\hline 13.1 & A & A & A & A & A & A & A & B & A & A \\
\hline 13.2 & A & A & A & A & A & A & A & A & A & A \\
\hline 14 & A & A & A & A & D & A & A & A & A & A \\
\hline 15 & A & A & B & A & A & A & A & A & A & A \\
\hline 16 & B & A & A & A & A & A & A & A & A & A \\
\hline 17 & A & B & D & B & A & B & A & A & A & C \\
\hline 18 & $\nabla$ & A & A & A & A & A & B & A & A & A \\
\hline 19 & A & A & B & A & A & A & A & A & A & A \\
\hline 20 & A & A & A & A & $\nabla$ & A & A & $\boldsymbol{\Delta}$ & A & A \\
\hline 21 & A & C & A & A & A & A & A & A & A & A \\
\hline 22 & A & A & A & B & A & A & A & B & A & A \\
\hline 23 & A & A & A & A & C & A & A & A & A & A \\
\hline 24 & A & A & B & A & A & A & A & B & A & A \\
\hline
\end{tabular}

A, B, C, D and E represented one, two, three, four and five band(s) respectively; $\boldsymbol{\Delta}$ represented the TCR V $\beta$ gene families which exhibited no band;

$\nabla$ represented the TCR V $\beta$ gene families which exhibited very blur band.

On the $12 \%$ polyacrylamide gel stained with silver solution, each picture of $\mathrm{V} \beta$ graph of the healthy volunteers also showed a thin band at about the position of $250 \mathrm{bp}$, and all like the picture of V $\beta 4$ of patient-1 (as shown as Figure 2), so the data was not shown as well. While for the patients with T1DM, except for most V $\beta$ genes exhibited single clear bands at the predicted position, several showed two or more than two bands, or there were a few genes which exhibited more blurry bands, even looked disappear (Figure 2, only the graph of patient-1 was shown). Compared to healthy volunteers, except for $\mathrm{V} \beta 10$ and V $\beta 13.2$, the other genes showed more or less skewed, the mainly biased genes also were V $\beta 7, \mathrm{~V} \beta 11$ and $\mathrm{V} \beta 17$, however, the bands of V $\beta 18$ of patient- 1 and $\mathrm{V} \beta 20$ of patient- 6 showed more blurry, that of V $\beta 20$ of patient-8 was deficient or deleted (Table 2).

Comparatively, the pictures attained by the silver staining technique were similar to that of the ethidium brominde one, however, the clarity of the bands of all the samples were different for two methods, it was better for the former than that of the latter; the number of the bands of several TCR V $\beta$ were not completely identical, generally, the number of the bands with the former technique was high than that with the latter one. For example, there were two and four blur bands in V $\beta 2$ and $\mathrm{V} \beta 7$ respectively in T1DM patient-1 detected by ethidium brominde staining technique, while by silver staining technique, there were four clear bands for $V \beta 2$, while the number for V $\beta 7$ was five (Figure 1 and Figure 2). We detected some of the $\mathrm{V} \beta$ families which exhibited more than two bands with the two methods, and found that the number and/or clarity of the bands assayed by silver staining technique were significantly higher and/or better than that by ethidium brominde one (Figure 3).

\section{Discussion}

Ethidium brominde staining technique is a classic method to detect expression of the RNA or DNA; recently, it has been used to quickly detect the clonality of TCR [11]. In the previous studies, we used the technique to detect the skewness of TCR $\mathrm{V} \alpha$ or/and $\mathrm{V} \beta$ genes of healthy human, patients with colorectal carcinoma, childhood patients with tuberculosis infections and children vaccinated with Bacillus Calmette-Guerin, and described the normal genes of health volunteers and biased gene families of some patients $[1,8,9]$, moreover, we found that the results detected with ethidium brominde staining technique were coincident with that with genescan or DNA melting curve analysis technique. This method was more quick and simple, so it can be taken as a proper technique to screen the skewness of TCR. However, because of its toxicity, its application is limited to some degree.

Silver staining technique is a sensitive method for detecting relative quantity of protein, enzyme, RNA, DNA and so on, which detectable range could be as low as the level of nanogram $[12,13]$. However, to date, if it can be used to detect the biases of TCR V genes remain unknown. In this study, we used the silver staining and ethidium brominde staining technique to assay the TCR V $\beta$ biases of T1DM patients simultaneously, and found that the TCR $\mathrm{V} \beta$ pictures of all ten patients detected with the former method were very similar to that of the latter one. V $\beta 7$, $\mathrm{V} \beta 11$ and $\mathrm{V} \beta 17$ were the mainly biased genes with higher frequencies, while V $\beta 10$ and V $\beta 13.2$ were the common genes which showed were not biased in all of the ten T1DM patients. There were also differences between the results detected by the two methods respectively. Firstly, in some TCR V $\beta$ genes of the same patients, the number of bands attained by silver staining technique was higher than that detected by ethidium brominde staining technique, such as V $\beta 2$ and V $\beta 7$ of patient-1, in the former method, the number were four and five respectively, while that were only two and four in the latter one. This indicated that silver staining technique could isolate the DNA more exactly than ethidium brominde staining technique. Secondly, the bands, especially besides the ones of about $250 \mathrm{bp}$, were much 
clearer with silver staining technique compared to that with ethidium brominde staining way, for example, at location of about 300bp, the band of TCR V $\beta 7$ of patient5 assayed by the former method was significantly obvious than which by the latter method (as showed in Figure 3). Thirdly, except for the electrophoresis instrument and staining reagents, silver staining technique needs no other equipment, while ethidium brominde technique needs the ultraviolet transilluminator to observe the results. Lastly, ethidium brominde dying is poisonous, and the ultraviolet ray is another harmful factor to researchers, while all of the instrument and reagents in the silver staining technique are safe to human user.

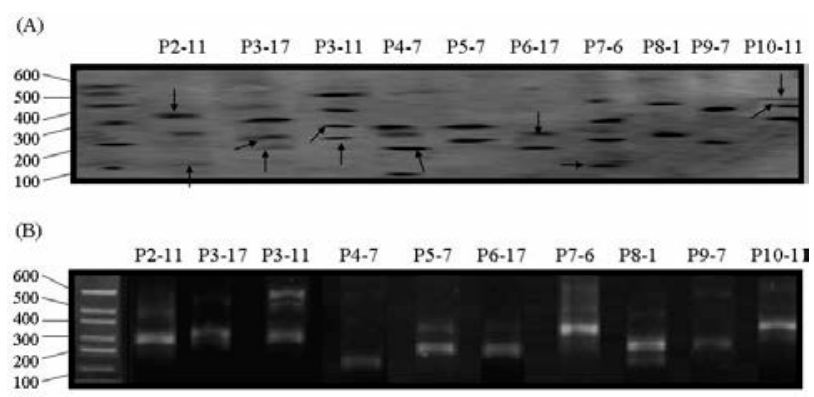

Figure 3. The comparative pictures for some of TCR V $\beta$ genes of TIDM patients which showed biased on $12 \%$ polyacrylamide gel by silver staining and on the $1.5 \%$ agarose gel by silver staining respectively. The number such as P2-11 on the figure represented as follows: P2-11: V $\beta 11$ of patient-2, P3-17: V $\beta 17$ of patient-3, P3-11: V $\beta 11$ of patient-3, P4-7: V $\beta 7$ of patient-4, P5-7: V $\beta 7$ of patient-5, P6-17: V $\beta 17$ of patient-6, P7-6: V $\beta 6$ of patient-7, P8-1: V $\beta 1$ of patient-8, P9-7: V $\beta 7$ of patient-9, P10-11: V $\beta 11$ of patient-10. (A) The graphs for some of TCR V $\beta$ genes of TIDM patients which showed biased on $12 \%$ polyacrylamide gel by silver staining. (B) The graphs for some of TCR V $\beta$ genes of TIDM patients which showed biased on the $1.5 \%$ agarose gel by ethidium brominde staining

However, the small sample size, the concentration of polyacrylamide gel and the relative complexity of the polyacrylamide gel electrophoresis are the main elements which affect the detection effect of silver staining technique; in future, we will use more cases and more appropriate conditions to explore the usage of the method in the fields of TCR skewness.

\section{Conclusion}

Overall, compared with ethidium brominde staining technique, silver staining is a quick, safe and effective method to detect the clonetypes of TCR V $\beta$ gene families of T1DM patients, it is worthy to consider as a screening assay to evaluate the bias of TCR V $\beta$.

\section{Acknowledgement}

All the authors thank the ten patients and volunteers for providing research samples, and the Science Research department of Jining Medical College and of the Affiliated Hospital for supporting this study. Subsequently, also thank the grant from the Provincial Science and Technology Development Project (No. 2012YD18054), the Provincial Nature Science Foundation (No. ZR2012HL29), the High School Science and Technology Plan Project (No. J11LF18), the Population and Family Planning Commission (No. [2011]13), and the Development Plan Project of Jining Science and Technology Bureau of Shandong Province (No. [2011]57), the Youth Foundation of Jining Medical College (No. [2011]).

\section{References}

[1] Zhou J, Ma R, Luo R, He X, Sun W, Tang W, Yao XS. Primary exploration of molecular and spectratyping features of CDR3 of TCR $\beta$ chain in the peripheral blood and tissue of patients with colorectal carcinoma. Cancer Epidemiology 2010, 34: 733-740.

[2] Uttenthal BJ, Chua I, Morris EC, Stauss HJ. Challenges in T cell receptor gene therapy. J Gene Med 2012, 14 (6):386-99.

[3] Frigstad T, Løset GÅ, Sandlie I, Bogen B. A Public T cell Receptor Recognized by a Monoclonal Antibody Specific for the D-J Junction of the $\beta$-chain. Scand J Immunol 2013, 78(4):345-51.

[4] Aung NY, Ohtake H, Iwaba A, Kato T, Ohe R, Tajima K, Nagase T, Yamakawa M. Angioimmunoblastic T-cell lymphoma with dual genotype of TCR and IgH genes. Pathol Res Pract 2011, 207(5):317-21.

[5] Kitaura K, Fujii Y, Matsutani T, Shirai K, Suzuki S, Takasaki T, Shimada S, Kametani Y, Shiina T, Takabayashi S, Katoh H, Ogasawara K, Kurane I,Suzuki R. A new method for quantitative analysis of the $\mathrm{T}$ cell receptor $\mathrm{V}$ region repertoires in healthy common marmosets by microplate hybridization assay. J Immunol Methods 2012, 384(1-2): 81-91.

[6] Van der Borght A, van der Aa A, Geusens P, Vandevyver C, Raus J, Stinissen P. Identification of overrepresented TCR genes in blood and tissue biopsies by PCR-ELISA. J Immunol Methods 1999, 223:47-61.

[7] Yao XS, Diao Y, Sun WB, Luo JM, Qin M, Tang XY. Analysis of the CDR3 length repertoire and diversity of TCR $\alpha$ chain in human peripheral blood T lymphocytes. Cell Mol Immunol 2007, 4:215-220.

[8] Zhou JW, Ma R, Tang WT, Lo R, Yao XS. Primary exploration of the third complementarity determining region spectratyping and molecular features of $\mathrm{T}$ cell receptor alpha chain in the peripheral blood and tissue of patients with colorectal carcinoma. ACTA Medica Mediterranea 2011, 27: 23-30.

[9] Zhou J, Kong C, Luo J, Cao J, Shi Y. Comparaing TCR beta chain variable gene skewness between children with tuberculosis and BCG-vaccinated children. Archive of Iranian Medicine 2013, 16:104108.

[10] Mberti KG, Zimmet PZ, Definition, diagnosis and classification of diabetes mellitus and its complications. Part 1: diagnosis and classification of diabetes mellitus provisional report of a WHO consultation. Diabet Med, 1998(15):539-53.

[11] Yao XS, Zheng XJ, Ma L, Sun WB, Zheng WY, Wang Q, Hu ZM, Meng MJ, Li Y, Wang ZQ, He XW, Wang JF, Wang XN. Analysis of the CDR3 region of alpha/ betaT-cell receptors (TCRs) and TCR $\mathrm{BD}$ gene double-stranded recombination signal sequence breaks end in peripheral blood mononuclear cells of T-lineage acute lymphoblastic leukemia. Clin Lab Haemat 2006, 28:405-415.

[12] Liu YB, Gao JH, Duan HJ. Detection and analysis of Fas gene (exon 1-6) mutation in keloids. Di Yi Jun Yi Da Xue Xue Bao 2002, 22(1): 61-63.

[13] Lendeckel U, Wex T, Arndt M, Frank K, Franke A, Ansorge S. Identification of point mutations in the aminopeptidase $\mathrm{N}$ gene by SSCP analysis and sequencing. Hum Mutat 1998, Supple 1: 158160. 